\title{
Fortalecimiento de la práctica docente con Learning Analytics: estudio de caso
}

\section{Lucy González Lerma}

Magíster en Informática Educativa Secretaria de Educación Distrital de Bogotá

https://orcid.org/0000-0001-9079-9052

lucygole@unisabana.edu.co

\section{Carlos Lugo Silva}

Doctor en Estudios Sociales de la Ciencia y la Tecnología Universidad de la Sabana

https://orcid.org/0000-0002-4312-8392 carloslugosilva@gmail.com

Artículo de Investigación

Recepción: 29 de abril de 2019

Aprobación: 30 de octubre de 2019 https://doi.org/10.19053/22160159.v11.n25.2020.9075

\section{Resumen}

Este artículo presenta el análisis y la evaluación de un estudio de caso sobre la implementación del software para la gestión de aula CloudLabs, realizado en una institución educativa de Bogotá, en el área de ciencias naturales en los grados de sexto a undécimo. El objetivo fue desarrollar la competencia analítica en los docentes mediante un ambiente de aprendizaje mixto, InnovalacTIC, para la apropiación de conocimientos y competencias en la personalización de los aprendizajes a partir de los datos. Desde una metodología cualitativa, centrada en un diseño de investigaciónacción, fue posible reconocer el aporte de las herramientas Learning Analytics y del laboratorio virtual CloudLabs en la personalización de aprendizajes $\mathrm{y}$ en la evaluación formativa. Asimismo, 
el ambiente de aprendizaje implementado permitió definir las competencias docentes requeridas y, en general, las dificultades que se deben superar para el aprovechamiento de los datos como herramienta de transformación en las aulas de clases.

Palabras clave: análisis de datos, aprendizaje, competencia docente, evaluación formativa, gestión de la información, TIC

\title{
Strengthening teaching practice with Learning Analytics: a case study
}

\begin{abstract}
This article shows the analysis and evaluation of a case study about the implementation of the CloudLabs classroom management software, conducted in an educational institution in Bogotá, in the area of natural sciences in sixth to eleventh grades. The objective was to develop the analytical competence in the teachers through a mixed learning environment, InnovalacTIC, for their appropriation of knowledge and skills in the personalization of learning on the basis of the data. From a qualitative methodology, focused on a researchaction design, it was possible to recognize the contribution of the Learning Analytics tools and of the CloudLabs virtual laboratory in the personalization of learning and in the formative evaluation. Moreover, the implemented learning environment allowed to define the required teaching competences and, in general, the difficulties that must be overcome for the use of data as a tool for transformation in classrooms.
\end{abstract}

Keywords: data analysis, learning, teacher competence, formative evaluation, information management, ICT

\section{Renforcement des pratiques des enseignants au moyen de L'Analytique de l'Apprentissage: une étude de cas}

\section{Résumé}

On présente l'analyse et l'évaluation d'une étude de cas sur la mise en place du logiciel CloudLabs pour la gestion de classe. L'étude a été effectuée dans un établissement éducatif à Bogotá 
dans le domaine des sciences naturelles pour les niveaux 6 à 11 de l'enseignement secondaire. L'objectif a été de développer des compétences analytiques chez les enseignants en instaurant un environnement d'apprentissage mixte visant à l'appropriation de connaissances et de compétences dans la personnalisation des apprentissages à partir des données. Sur la base d'une méthode qualitative centrée sur une conception de recherche-action, il a été possible de reconnaître l'apport des outils de L'Analytique de l'Apprentissage et du laboratoire virtuel CloudLabs à la personnalisation des apprentissages et à l'évaluation formative. De même, l'environnement d'apprentissage mis en œuvre a permis d'identifier les compétences des enseignants qui sont requises et, en général, les difficultés qu'ils doivent surmonter pour utiliser les données comme un outil de transformation dans les salles de classe.

Mots-clés : analyse des données, apprentissage, compétences des enseignants, évaluation formative, gestion de l'information, TIC

\section{Fortalecimento da prática docente com o Learning Analytics: estudo de caso}

\section{Resumo}

Mostra-se a análise e avaliação de um estudo de caso sobre implementação do software para a gestão da sala CloudLabs, realizado numa instituição educativa em Bogotá, na área de ciências naturais nas séries de sexto ao décimo primeiro. $\mathrm{O}$ objetivo foi desenvolver a competência analítica em docentes através de um ambiente de aprendizagem misto, InnovalacTIC, para a apropriação de conhecimentos e competências na personalização de aprendizagem a partir dos dados. Desde de uma metodologia qualitativa, centrada em um desenho de pesquisa-ação, foi possível reconhecer a contribuição das ferramentas de Learning Analytics e do laboratório virtual CloudLabs na personalização de aprendizagem e na avaliação formativa. Assim mesmo, o ambiente de aprendizagem implementado permitiu definir as competências docentes requeridas e, em geral, as dificuldades que devem superar para o aproveitamento dos dados como ferramenta de transformação nas salas de classe.

Palavras-chave: análise de dados, aprendizagem, competência docente, avaliação formativa, gestão da informação, TIC 


\section{Introducción}

El crecimiento exponencial de las tecnologías de la información y la comunicación [TIC] y el incremento de las velocidades de internet han impulsado la propagación de nuevas herramientas que se complementan con las metodologías de aprendizaje y la transformación de la didáctica. De acuerdo con la Unesco (2013), las TIC ofrecen múltiples respuestas a las necesidades de aprendizaje, al desarrollo de las habilidades docentes, a la calidad del aprendizaje y a la gestión de la educación; siempre y cuando se utilicen bajo una intencionalidad pedagógica contundente, capaz de responder a la exigencia del mundo actual.

De acuerdo con la Cumbre Mundial sobre la Sociedad de la Información (Betancourt, 2004), el rápido progreso de las TIC genera oportunidades a las sociedades para alcanzar altos niveles de desarrollo, al considerar el impacto del acceso a la información y a la construcción del conocimiento. Esto expresa los beneficios esperados en el escenario pedagógico a través de los aportes de la innovación educativa de las denominadas tecnologías emergentes.

Es así como las instituciones educativas se han visto convocadas a responder las necesidades y mejorar los resultados académicos y de gestión escolar por medio de las TIC para el análisis de datos de forma masiva (Chiappe \& Rodríguez, 2017). No obstante, este proceso exige la preparación del docente y la consolidación de habilidades para el empleo de herramientas y el tratamiento masivo de la información, a fin de cualificar el aprendizajey ofrecer diferentes perspectivas sobre el desarrollo de su competencia analítica.

Ante este panorama, se planteó una investigación cuyo interés fue estudiar la apropiación de Learning Analytics a través de un gestor de aula y el impacto de CloudLabs en la práctica pedagógica, con la simulación de laboratorios virtuales. El uso de esta herramiente en el aula implica "la interpretación de un amplio rango de datos producidos y recogidos sobre los estudiantes, para orientar a docentes y gestores de la educación, sobre su progresión académica, para predecir actuaciones futuras e identificar elementos problemáticos" (Concari, 2014, p. 6).

Por esta razón, para llevar a cabo el proceso de seguimiento de aprendizajes de los estudiantes, el docente requiere desarrollar la competencia analítica, que se complementa con competencias 
de tipo pedagógico, tecnológico, comunicativo, investigativo y de gestión. En este sentido, el estudio se orientó hacia la consolidación de estrategias didácticas para impactar en los resultados académicos de los estudiantes y en el acompañamiento personalizado de su ruta de aprendizaje, desde el uso de una herramienta tecnológica para producir una evaluación acorde con los objetivos de aprendizaje.

En busca de mejorar el proceso pedagógico a través de las tecnologías emergentes, se planteó la siguiente pregunta: ¿cómo el gestor de aula del laboratorio virtual CloudLabs contribuye a los docentes de ciencias naturales de los grados de sexto a undécimo, para el seguimiento del aprendizaje y la evaluación formativa de los estudiantes, mediante la analítica de datos?

\section{Antecedentes}

Con el propósito de conocer las herramientas didácticas para el desarrollo de las competencias docentes, se rastrearon investigaciones de los ámbitos internacional, nacional y local, desde las categorías relacionadas con herramientas analíticas, educación con Learning Analytics y el papel de las familias en la educación personalizada. $\mathrm{Al}$ respecto, diferentes autores (Blikstein \& Worsley, 2016; Chiappe y Rodríguez, 2017; Shute \& Rahimi, 2017) señalan la importancia de la analítica de datos para la comprensión del aprendizaje de manera multimodal, que amplía el panorama hacia nuevas formas de evaluación.

Inicialmente, el trabajo de Esteve-Mon, Gisbert y Lázaro (2016) plantea que el rol del docente actual es capacitar a los estudiantes por medio de todas las potencialidades que ofrecen las TIC. Estos autores proponen que los docentes tengan una alfabetización digital básica y estén en la capacidad de integrarlas en sus prácticas pedagógicas, para lo cual su formación digital resulta fundamental. Por su parte, Badia, Chumpitaz, Vargas y Suárez (2016) identificaron tres grupos de competencias en los docentes, definidas como básicas, pedagógicas y tecnológicas.

Ahora bien, de acuerdo con Hung (2015), la consolidación de investigaciones y de propuestas que promuevan el uso de Learning Analytics para la mejora de la calidad educativa tiene un carácter incipiente en Colombia. Por eso, es importante dar a conocer algunas experiencias que ilustran los avances sobre el uso de esta 
herramienta para la analítica de datos en el aula, en consideración de aquellas prácticas significativas que fueron identificadas en la búsqueda.

\section{Herramienta analítica para evaluar el aprendizaje}

El trabajo realizado por Shute y Rahimi (2017) presenta cómo la evaluación basada en computadora para el aprendizaje permite desarrollar las competencias de los estudiantes de primaria y secundaria. Desde la revisión de diferentes herramientas para el análisis del aprendizaje, afirman que la retroalimentación, para ser más beneficiosa, no debe ser demasiado compleja y debe utilizarse de manera efectiva, centrada en respaldar la aprehensión del conocimiento y el fortalecimiento de habilidades de pensamiento.

Por otro lado, en el contexto local se está gestando un proyecto piloto con cinco colegios oficiales, mediante el uso de la plataforma de notas Apoyo escolar, que ofrece gratuitamente la Secretaría de Educación de Bogotá [SED] por medio de la Oficina Tecnologías y Sistemas de Información REDP. El proyecto consiste en la implementación de las herramientas de análisis de datos y presentación de informes Microsoft Power BI. Estos reportes pueden ser usados por rectores y coordinadores.

\section{Educando con Learning Analytics}

Un caso que ilustra las herramientas con las que un profesor puede aplicar Learning Analytics y el tiempo que requiere para lograrlo es el trabajo de Amo y Rodríguez (2017) titulado Educando con Learning Analytics. Aquí se da a conocer una entrevista efectuada al profesor Iñaki Fernández de la asignatura de biología en secundaria y responsable de innovación tecnológica en el Colegio Real Monasterio - Barcelona-. A partir de ahí, se pudo observar cómo el análisis de datos ayuda no solamente a mejorar los desempeños académicos de los estudiantes, quienes pueden averiguar en qué fallan, sino también al proceso pedagógico.

Desde esta experiencia se comprende que el docente posee datos concretos para asesorar a los estudiantes, quienes realizan un proceso metacognitivo para mejorar su aprendizaje. También muestra la importancia de la disposición del docente hacia el uso de la analítica de datos como una herramienta válida para orientar 
la búsqueda de estrategias efectivas para mejorar los procesos de enseñanza y aprendizaje.

\section{Las familias en la personalización de la educación}

Un aspecto importante en la investigación fue la personalización dela educaciónyel papel fundamental dela familia en la consolidación de estos procesos. Al respecto, la SED puso a disposición la Guía metodológica para el fortalecimiento de la alianza familiaescuela (2018a), para orientar a los padres, profesores y directivos docentes, y contribuir de forma significativa a mejorar las prácticas de aprendizaje. Con esta guía se busca afianzar la relación familiaescuela, con acciones y estrategias que les permitan reflexionar, resignificar y transformar los procesos educativos de los estudiantes.

Dichas experiencias investigativas marcaron un camino teórico para esta investigación, en la medida en que permitieron comprender la importancia de implementar las tecnologías emergentes para atender a las dificultades de cada alumno y, con esto, reforzar los aprendizajes construidos en el aula.

\section{Ámbito y delimitación teórica}

La investigación se enfocó en dos referentes que constituyeron el horizonte conceptual que orientó el estudio: por un lado, referentes tecnológicos, desde los conceptos de Learning Analytics, laboratorio virtual, gestor de aula y competencias TIC; por otro, los referentes pedagógicos constituidos en la evaluación formativa, el seguimiento, la personalización, las estrategias de aprendizaje y la seguridad de datos.

\section{Referentes tecnológicos}

\section{Learning Analytics.}

La herramienta de Learning Analytics busca personalizar la educación, al considerar el potencial, las dificultades y los avances de los estudiantes. Eso implica adaptar las actividades, contenidos, metodologías y didácticas en las prácticas de enseñanza, con el fin de permitir al estudiante avanzar en su proceso académico, teniendo en cuenta que, en opinión de Siemens y Long (2011), esta forma de comprender el aprendizaje involucra "el uso de datos inteligentes, datos producidos por el aprendiz y modelos de análisis para 
descubrir información y conexiones sociales, predecir y asesorar sobre el proceso" (p. 34).

Dos conceptos que introduce la investigación son Teaching y Learning Analytics. Teaching Analytics tiene el potencial de analizar diseños educativos, conformados por actividades de aprendizaje, prácticas de evaluación, recursos y herramientas. Learning Analytics está centrado en la medición, recopilación y análisis de los datos educativos de los alumnos en el contexto de aprendizaje. Ambos términos representan un campo de investigación que, según la revisión de literatura, ha generado beneficios para el diseño educativo, la investigación sobre la práctica docente y el seguimiento puntual a los resultados de los alumnos.

Justamente, es aquí donde, sin importar el nivel de profundidad de los análisis o del enfoque aplicado, surge un fundamento teórico clave para el mayor aprovechamiento de las tecnologías de análisis: las teorías de aprendizaje. En opinión de Wong et al. (2019), estas tecnologías orientan las decisiones sobre los tipos de datos que se recopilarán y los enfoques de análisis a utilizar, de acuerdo con las necesidades particulares del proceso.

Es así como las teorías desempeñan un papel imprescindible frente a la explicación del concepto de aprendizaje que se está midiendo con el dato. Este fue el caso de los docentes que utilizaron la plataforma del gestor de aula CloudLabs en Bogotá, quienes enriquecieron su trabajo al aplicar la teoría del aprendizaje constructivista y darle un sentido a la práctica con Learning Analytics, que, por ende, generó un impacto en los resultados del diseño de itinerarios para responder a los estilos de aprendizaje de los estudiantes.

\section{Laboratorio virtual}

Los laboratorios virtuales como herramienta de aprendizaje TIC permiten realizar las prácticas a través de la simulación, en la medida que funcionan como una alternativa a los laboratorios convencionales. Según García (2016), estos laboratorios son una ventaja para estudiantes y docentes, al facilitar la enseñanza y permitir la comprensión de temas relacionados con las ciencias naturales, la física y la química, el medio ambiente y la seguridad. Por eso, su característica más destacable es permitir "el desarrollo de 
prácticas educativas que conlleven al educando a comprender mejor su entorno a través de prácticas sencillas y seguras que permitan afianzar lo visto en clase, con la praxis de un laboratorio simulado" (p. 15).

\section{Gestor de aula}

Es un conjunto de herramientas similares a los sistemas de gestión de aprendizaje, para realizar intervenciones en tiempo real por parte de estudiantes, profesores e institución educativa, con el propósito de seguir el avance del aprendizaje y evaluar su progreso para mejorar la calidad de la enseñanza. Una vez se generan los reportes, tanto a nivel individual como grupal, se pueden establecer el dominio de los temas, las dificultades y las comparaciones con las clases de aula. $\mathrm{Al}$ respecto, autores como Siemens y Long (2011) aseguran que:

Los datos generados por el alumno son una valiosa fuente de información sobre lo que está sucediendo en el proceso de enseñanzaaprendizaje y sugieren formas en que los educadores pueden realizar mejoras al proceso, de manera que aumenten los niveles de comprensión, interés y demás aspectos que sean benéficos para el estudiante. También este análisis puede dar un panorama sobre los estudiantes que están en peligro de deserción escolar o requieren apoyo para aumentar su éxito. (p.32)

\section{Competencias TIC}

Uno de los principales referentes para el estudio propuesto son las competencias TIC para el desarrollo profesional docente. Durante la última década, las competencias han sido el eje articulador del sistema educativo de Colombia. Son entendidas como "el conjunto de conocimientos, habilidades, actitudes, comprensiones $\mathrm{y}$ disposiciones cognitivas, socioafectivas $\mathrm{y}$ psicomotoras apropiadamente relacionadas entre sí para facilitar el desempeño flexible, eficaz y con sentido de una actividad en contextos relativamente nuevos y retadores" (Vasco, citado por Ministerio de Educación Nacional [MEN], 2006, p. 2).

En desarrollo profesional docente y la innovación educativa se concentra un conjunto de competencias que contribuyen al enriquecimiento del aprendizaje y a la adopción de estrategias para la transformación del entorno (MEN, 2013). Entre estas competencias se destacan: las competencias tecnológica, pedagógica y analítica, 
y las competencias de gestión, comunicación e investigación, apoyadas en el uso de las TIC (Niño \& Ariza, 2015). En la figura 1 se resumen las características de cada una y su aporte a la investigación planteada.

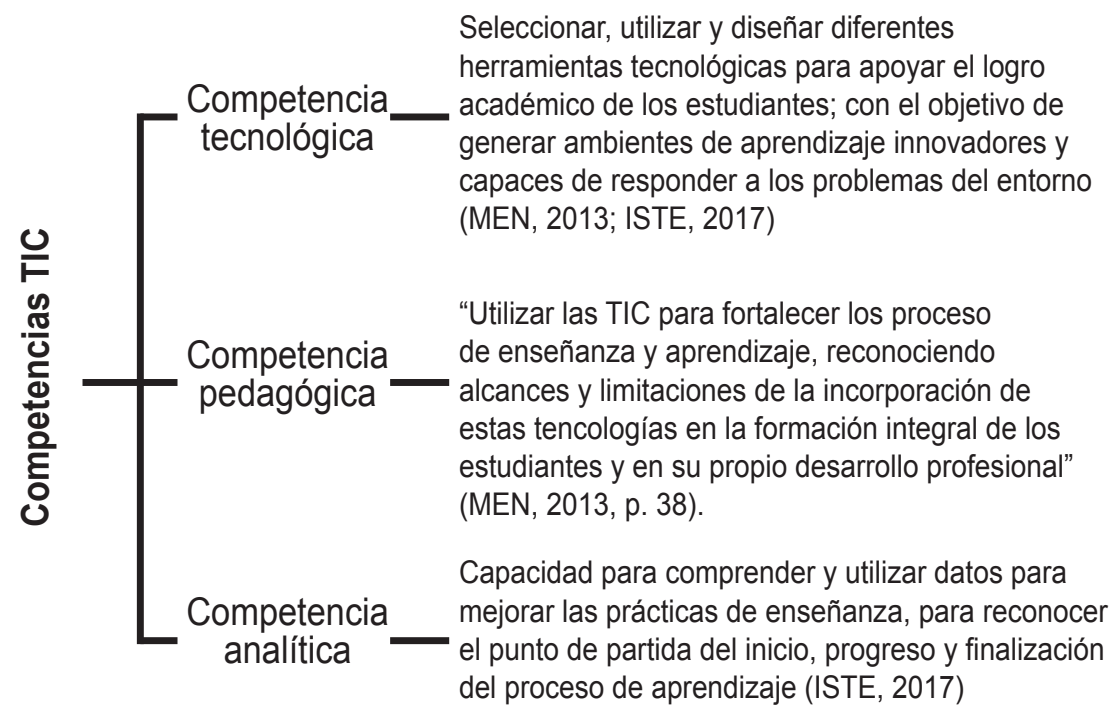

Figura 1. Competencias TIC docentes. Fuente: elaboración propia basada en MEN (2013) e International Society for Technology in Education [ISTE] (2017).

\section{Competencia analítica}

La nueva ciudadanía digital y la propuesta del rol docente aparecen definidas en los estándares de la ISTE, donde se incluyó el desarrollo de la competencia analítica en los docentes como elemento fundamental para apropiarse de metodologías y plataformas Learning Analytics. Sin embargo, en la última versión 2019 del estándar del ISTE, se crea una competencia específica "analista", en la cual se desarrollan los siguientes indicadores (tabla 1). 


\section{Tabla 1}

\section{Competencia analista docente}

\begin{tabular}{cl}
\hline \multicolumn{1}{c}{ Analista } \\
\hline $\begin{array}{l}\text { Docentes que comprenden y utilizan datos para mejorar la enseñanza y apoyar } \\
\text { a sus estudiantes en el logro de sus objetivos de aprendizaje. Para esto, los } \\
\text { docentes deben: }\end{array}$ \\
\hline 7a & $\begin{array}{l}\text { Proporcionar formas alternativas para que los estudiantes } \\
\text { demuestren competencia y reflexionar sobre su aprendizaje } \\
\text { utilizando la tecnología. }\end{array}$ \\
& $\begin{array}{l}\text { Usar la tecnología para diseñar e implementar una variedad de } \\
\text { contenidos y evaluaciones que satisfacen las necesidades de los }\end{array}$ \\
estudiantes. Proporcionar comentarios oportunos a los estudiantes \\
e informar a la instrucción.
\end{tabular}

Fuente: ISTE (2019).

\section{Referentes pedagógicos}

\section{Evaluación formativa}

La implementación de herramientas para la analítica de datos tiene impacto directo en la evaluación formativa o evaluación para el aprendizaje. Sin duda, la herramienta Learning Analytics desempeña un papel importante en el ejercicio evaluativo, en cuanto es un recurso tecnológico a disposición del docente para analizar la apropiación de las competencias de los estudiantes, relacionadas con el conocimiento adquirido. Es así como la evaluación, mediada por este tipo de herramientas tecnológicas, permite realizar los ajustes que requieren los estudiantes para satisfacer sus necesidades formativas.

De acuerdo con Díaz y Hernández (2002), la finalidad de esta forma de evaluación es "estrictamente pedagógica, regular el proceso enseñanza aprendizaje, para adaptar o ajustar, las condiciones pedagógicas (estrategias, actividades) en servicio del aprendizaje de los alumnos" (p. 406). Learning Analytics brinda a los docentes la oportunidad de leer y analizar esos datos generados durante el proceso de aprendizaje y en las evaluaciones individuales o grupales, para retroalimentar todo el trabajo realizado. 


\section{Seguimiento de aprendizajes}

Es la acción de identificar los factores que originan el aprendizaje con éxito y, a su vez, las dificultades para ejercer la medición y seguimiento, el cual es un ejercicio clave, en cuanto influye en la conducta, las formas y estilos de aprendizaje que se presentan dentro de un aula.

\section{Estrategias}

Esta investigación es abordada desde el constructivismo y el aprendizaje significativo social, independiente y autónomo, mediante la consolidación de ambientes de aprendizaje y estrategias que contribuyan a este proceso, tales como: estrategias que activan los conocimientos previos, orientan y guían los aprendizajes, y organizan la información nueva por aprender. De manera que, según Díaz y Hernández (2002), en este marco pedagógico "la enseñanza corre a cargo del enseñante como su originador; pero al fin y al cabo es una construcción conjunta como producto de los continuos y complejos intercambios con los alumnos y el contexto instruccional" (p. 143).

\section{Personalización del aprendizaje}

De acuerdo con la Unesco (2017), el aprendizaje personalizado consiste en "prestar especial atención a los conocimientos previos, las necesidades, las capacidades y las percepciones de los estudiantes durante los procesos de enseñanza y aprendizaje. Se trata, por lo tanto, de una formación centrada en el alumno" (p. 5). Esto implica que el alumno asuma un rol activo en el proceso, mientras que el docente es el que guía y orienta los contenidos, de acuerdo con los ritmos de aprendizaje de sus estudiantes. Así la personalización del aprendizaje surge con la identificación por parte del docente de los conocimientos, las competencias, las conductas y los enfoques contenidos en el programa de estudios, de forma que propicie el interés de los estudiantes y les resulten valiosos, comprensibles y pertinentes.

\section{Seguridad de los datos}

La seguridad de datos fue un componente relevante en esta investigación, teniendo en cuenta que Learning Analytics implica el uso de un alto volumen de datos, entre los que se encuentran big data, que, por tratarse de datos personales, son susceptibles de ser 
analizados bajo las normas que los regulan. En Colombia se dispuso la Ley estatutaria 1581 "protección de datos personales" (Congreso de Colombia, 2012) y la Resolución 1944 "política de seguridad de la información” (SED, 2017). También está la Resolución 1739, la cual adopta la política de tratamiento de datos personales en la SED (2018c).

Desde los referentes teóricos y pedagógicos abordados fue posible reflexionar sobre la importancia de generar prácticas mediadas por el uso de las TIC y la apropiación de herramientas virtuales y gestores de aula. En esta medida, el panorama descrito ofrece la oportunidad de comprender los aspectos pedagógicos que implica Learning Analytics, dentro del diseño de propuestas pedagógicas más efectivas.

\section{Metodología}

La investigación se basó en una metodología de tipo cualitativo, centrada en un estudio de caso mediante la observación y reflexión del proceso de enseñanza y aprendizaje de los docentes. En este enfoque de investigación la recolección de los datos se realiza desde las perspectivas y opiniones de los participantes, teniendo en cuenta sus experiencias y formas de interactuar en determinados contextos (Hernández, Fernández \& Baptista, 2006).

El alcance de la investigación se limitó al análisis de la implementación de las aplicaciones propuestas, el desarrollo de competencias docentes y los efectos en la práctica pedagógica, a través del desarrollo de una micropráctica en un caso específico. Para el diseño se retomaron algunos de los postulados de la investigaciónacción, como una oportunidad de relacionarse con las problemáticas y prácticas cotidianas que experimentan los docentes en el aula.

La investigación se realizó en un colegio oficial de Bogotá. De las 382 instituciones distritales, 40 fueron seleccionadas por el proyecto Saber Digital de la SED (2018b) para trabajar con docentes de ciencias naturales, física y química en las aplicaciones de laboratorios virtuales CloudLabs, adquiridas por el Ministerio de las TIC. De entre ellas fue seleccionado un colegio para hacer el estudio de caso con el uso de CloudLabs y su gestor de aula, donado por la empresa Innove Education, que operó como patrocinadora de este paquete de herramientas. 
Para esto, se llevó a cabo una práctica específica enfocada en el seguimiento de los aprendizajes y la evaluación formativa de los estudiantes. Uno de los procedimientos fue el uso del laboratorio virtual CloudLabs. Como evidencia de estas prácticas se generaron reportes que alimentaron el gestor de aula, encargado de procesar y consolidar los datos académicos. Además, el docente pudo realizar consultas individuales y grupales que fueron analizadas, a favor de los aprendizajes y las competencias.

De igual manera, se hicieron un seguimiento, observación y acompañamiento a las prácticas de un grupo de docentes del área de ciencias naturales de la institución educativa, donde fueron asignadas 40 licencias para seis profesores, distribuidas así: 20 para el área de química, 10 para física y 10 para ciencias naturales, lo que determinó la forma como se trabajó con los estudiantes, el seguimiento de los aprendizajes y su correspondiente evaluación.

Ahora bien, el diseño tecnopedagógico implementado para el proceso se basó en un enfoque pedagógico constructivista, donde el docente realiza una apropiación directa de las herramientas de tecnología emergente (Díaz \& Hernández, 2002). Para esto se construyó un ambiente de aprendizaje titulado InnovalacTIC Innova con Learning Analytics y TIC-, con el apoyo de un aula virtual alojada en la plataforma Moodle de la SED ${ }^{1}$. En la tabla 2 se detallan los objetivos, actividades y recursos utilizados para el ambiente de aprendizaje propuesto.

Tabla 2

Descripción del ambiente de aprendizaje: InnovalacTIC

\begin{tabular}{|c|c|}
\hline \multicolumn{2}{|r|}{ Ambiente de aprendizaje: InnovalacTIC } \\
\hline Objetivo & $\begin{array}{l}\text { Integrar el laboratorio virtual y gestor del aula en las prácticas } \\
\text { docentes mediante un seguimiento a los aprendizajes y evaluación } \\
\text { formativa de estudiantes. }\end{array}$ \\
\hline $\mathrm{O}$ & $\begin{array}{l}\text { - Identificar los conceptos y el manejo del gestor de aula, } \\
\text { laboratorio virtual y analíticas de datos. }\end{array}$ \\
\hline específicos & $\begin{array}{l}\text { - Desarrollar actividades y micropráctica por parte de los docentes } \\
\text { integrando el gestor de aula y el laboratorio para el seguimiento } \\
\text { de los aprendizajes y la evaluación formativa de sus estudiantes. }\end{array}$ \\
\hline
\end{tabular}

$1 \quad$ El ambiente de aprendizaje está alojado en las aulas virtuales de la Red Académica de la SED, disponible en https://aulasvirtuales.redacademica.edu.co/moodle/ course/view.php?id=6\&section $=1$ 


\begin{tabular}{|c|c|c|c|}
\hline Contenidos & $\begin{array}{l}\text { - Presentación d } \\
\text { - Competencias } \\
\text { - Tema 1: labora } \\
\text { - Tema 2: gestor }\end{array}$ & $\begin{array}{l}\text { ambiente de aprendizaje. } \\
\text { ocentes. } \\
\text { rio virtual. } \\
\text { le aula, Learning Analytics. }\end{array}$ & \\
\hline Recursos & \multicolumn{3}{|c|}{$\begin{array}{l}\text { Computadores, televisor, aplicaciones, laboratorio virtual y gestor } \\
\text { de aula, internet, manuales de las aplicaciones, herramientas TIC. }\end{array}$} \\
\hline \multicolumn{4}{|c|}{ Estrategia pedagógica: enseñanza estratégica } \\
\hline \multicolumn{2}{|c|}{$\begin{array}{l}\text { Preparación para el } \\
\text { aprendizaje }\end{array}$} & $\begin{array}{l}\text { Presentación de contenidos } \\
\text { que se van a aprender }\end{array}$ & $\begin{array}{l}\text { Aplicación e } \\
\text { integración }\end{array}$ \\
\hline \multicolumn{2}{|c|}{$\begin{array}{l}\text { Uso de conocimientos previos } \\
\text { para promover nuevos } \\
\text { aprendizajes }\end{array}$} & $\begin{array}{l}\text { Mediante el uso de } \\
\text { infografías, videos, } \\
\text { presentación Sway y } \\
\text { manuales. }\end{array}$ & $\begin{array}{l}\text { Actividades } \\
\text { propuestas y } \\
\text { micropráctica de } \\
\text { personalización } \\
\text { de aprendizajes. }\end{array}$ \\
\hline
\end{tabular}

Fuente: elaboración propia.

En este ambiente de aprendizaje B-Learning los datos se recogieron con instrumentos como diario de campo, entrevistas y apoyo con las actividades propuestas a los docentes para integrar el gestor de aula y el laboratorio virtual, en el seguimiento y evaluación de los estudiantes. Antes de la implementación, se llevó a cabo un pilotaje con docentes de otra institución, donde se enfatizó en la revisión del diseño general de contenidos, especialmente el tema de Learning Analytics, puesto que este colegio contaba con el laboratorio, mas no con el gestor.

También se evidenció, como resultado del diagnóstico y de la observación con registro en un diario de campo, el desconocimiento de Learning Analytics y de la analítica de datos, tanto en profesores del pilotaje como en el grupo focal. En la identificación de las aplicaciones laboratorio virtual CloudLabs, gestor de aula y Learning Analytics, se realizó una capacitación de 16 horas presenciales y 24 virtuales, con los docentes del grupo focal. La segunda fase experiencial se enfocó en el uso de las aplicaciones y su relación con Learning Analytics, mediante el desarrollo de actividades que permitieran responder a la pregunta de investigación.

En el desarrollo de la fase práctica se presentaron algunas dificultades de tipo técnico como fallas de computadores e internet. Algunos docentes lograron superarlas, otros continuaron gestionando la solución con apoyo de la investigadora ante la oficina 
de las tecnologías de la SED. Se lograron mejoras en el servicio, tales como anchos de banda en internet de 30 a 60 Mbps y estrategias de la mesa de ayuda con visitas periódicas a los colegios.

\section{Desarrollo de las actividades}

Para alcanzar los objetivos específicos de la investigación, se propusieron a los docentes participantes cuatro actividades contenidas en dos temas, distribuidas en la tabla 3.

Tabla 3

Temas y actividades del AA: InnovalaTIC

\begin{tabular}{|c|c|c|}
\hline \multicolumn{3}{|c|}{ Temas y actividades del AA InnovalaTIC } \\
\hline Tema & Actividad & Objetivo \\
\hline $\begin{array}{l}\text { Tema 1: simulación } \\
\text { mediante el uso del } \\
\text { laboratorio virtual }\end{array}$ & $\begin{array}{l}\text { 1. Foro: uso y beneficio } \\
\text { de los laboratorios } \\
\text { virtuales }\end{array}$ & $\begin{array}{l}\text { Identificar los beneficios de } \\
\text { los laboratorios CloudLabs } \\
\text { en el seguimiento de los } \\
\text { aprendizajes, personalización } \\
\text { de los aprendizajes, así como las } \\
\text { diferencias con el laboratorio } \\
\text { físico }\end{array}$ \\
\hline \multirow{3}{*}{$\begin{array}{l}\text { Tema 2: Learning } \\
\text { Analytics }\end{array}$} & $\begin{array}{l}\text { 1. Trabajo } \\
\text { independiente } \\
\text { Learning Analytics } \\
\text { desde el gestor }\end{array}$ & $\begin{array}{l}\text { Reflexionar sobre los beneficios } \\
\text { del gestor como herramienta } \\
\text { para aplicar Learning Analytics }\end{array}$ \\
\hline & $\begin{array}{l}\text { 2. Micropráctica } \\
\text { Learning Analytics }\end{array}$ & $\begin{array}{l}\text { Aplicar Learning Analytics } \\
\text { proponiendo tres estrategias de } \\
\text { personalización del aprendizaje } \\
\text { para sus estudiantes, con base en } \\
\text { los reportes del gestor de aula. }\end{array}$ \\
\hline & $\begin{array}{l}\text { 3. Foro reflexión: ¿̇qué } \\
\text { competencias TIC } \\
\text { requiere el docente? }\end{array}$ & $\begin{array}{l}\text { Reflexionar sobre las } \\
\text { competencias que está } \\
\text { desarrollando el docente al } \\
\text { integrar en sus prácticas el } \\
\text { uso de laboratorios virtuales, } \\
\text { el gestor de aula y Learning } \\
\text { Analytics. }\end{array}$ \\
\hline
\end{tabular}

Fuente: elaboración propia.

Para el desarrollo del tema 1, el docente participante elaboró un documento donde estableció los aportes que genera el laboratorio virtual en el aula de clase. Asimismo, se preguntó sobre los beneficios en su disciplina académica y las diferencias de la práctica virtual -laboratorio virtual-, teniendo en cuenta las características del laboratorio convencional presencial. 
En la actividad 1 del tema 2, los docentes diseñaron un documento donde expusieron la contribución del gestor de aula a sus prácticas pedagógicas. En la actividad 2 se propuso una micropráctica, mediante los reportes del gestor de aula generados desde las prácticas de laboratorios virtuales de los estudiantes. Con el análisis de los reportes, se propuso al docente un formato planeador didáctico, con el que diseñó una clase para tres tipos de necesidades educativas, según los siguientes criterios de clasificación de sus estudiantes: avanzado -desempeño mayor al necesario-, medio desempeño acorde al esperado-, bajo - requiere refuerzo-.

En la actividad 3, se planteó el objetivo de reflexionar sobre las competencias que requiere o está desarrollando el docente al integrar en sus prácticas el uso de laboratorios virtuales, el gestor de aula y Learning Analytics. Para esto se dispuso una presentación sobre las competencias TIC para el desarrollo profesional docente. Al inicio del proceso de capacitación se realizó el diagnóstico relacionado con las competencias propuestas por el MEN (2013). Una vez finalizadas las actividades, el docente debía opinar sobre las competencias que implica integrar en sus prácticas el uso de laboratorios virtuales, el gestor de aula y Learning Analytics, para reflexionar si las competencias sugeridas por el MEN eran suficientes o si requerían de otras.

\section{Codificación y análisis}

El proyecto comprendió el uso de la herramienta Qda miner para la codificación y análisis de las entrevistas y diarios de campo de observación. Es importante establecer que para este proceso, como producto del marco teórico y los objetivos planteados, se plantearon dos categorías relacionadas con tecnologías emergentes TIC y prácticas pedagógicas. Dela primera se desprenden las subcategorías laboratorio virtual, gestor de aula y Learning Analytics, como un apoyo educativo al quehacer de los docentes en el aula. En cuanto a la segunda categoría, se desprenden las subcategorías estrategias pedagógicas, seguimiento de aprendizajes, evaluación formativa y, por último, didácticas (figura 2). 


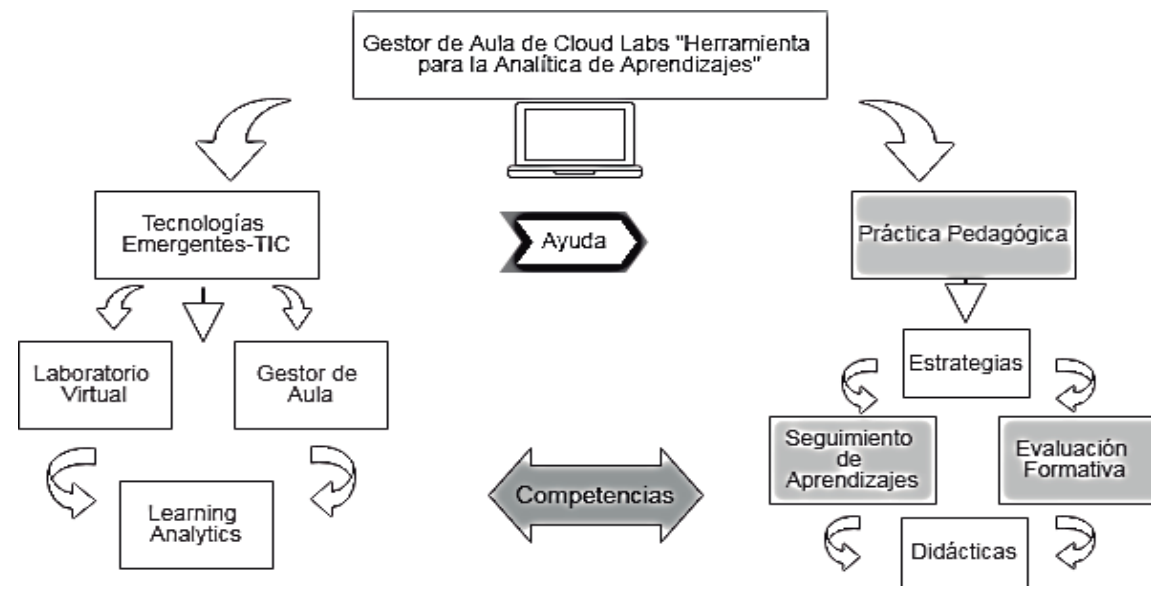

Figura 2. Categorías propuestas y emergentes. Fuente: elaboración propia.

\section{Resultados}

\section{Actividades}

Los primeros resultados -ya publicados (Lugo y González, 2018) - permitieron conocer las percepciones iniciales de los docentes frente a la analítica de datos, así como las barreras de entrada en la implementación de herramientas de análisis de esta información. Con esto fue posible determinar lo que opinan los docentes sobre la analítica: "es un ejercicio que requiere de competencias muy avanzadas", "requiere de la capacitación previa" "son proyectos de más carga administrativa".

Sin duda, esto respondió al principio de la investigación-acción centrado en contemplar los problemas desde el punto de vista de quienes están implicados en este, validado a partir de un diálogo libre y espontáneo sobre el ejercicio realizado en la investigación. A partir de ahí, se vio la necesidad de atender a los docentes desde una capacitación constante, acompañamiento y actualización, como parte del accionar para fortalecer los procesos pedagógicos basados en la propuesta ISTE (2017).

De igual forma, para superar la falta de interés por estas nuevas pedagogías y herramientas, así como el desconocimiento de este tipo de plataformas y herramientas analíticas, los resultados indicaron la importancia de generar una cultura digital, apropiar los beneficios 
de estas tecnologías y avanzar en el desarrollo de las competencias analíticas, las habilidades psicoeducativas y de liderazgo (Lugo y González, 2018).

Una vez realizado este acercamiento inicial, se presentó un esquema con los resultados obtenidos del laboratorio virtual y el gestor de aula de CloudLabs a partir de la actividad 1 de los temas 1 y 2 (tabla 2), donde se realizó un seguimiento detallado de los resultados y progresos en los aprendizajes de los estudiantes. De ahí se obtuvo que fue posible el trabajo en equipo ante diferentes niveles, capacidades y discapacidades, en consideración de las inquietudes, las motivaciones de los estudiantes y la observación de los ritmos de aprendizaje.

También surgió la discusión en relación con los procesos observados y las experiencias de los estudiantes, lo que en cierto modo aportó a la praxis en la aplicación de estrategias pertinentes para clases. Entonces, el trabajo en equipos de estudiantes, el seguimiento personalizado y contribución al desarrollo de las metodologías participativas se presentan como los aportes más significativos del gestor de aula (figura 3).

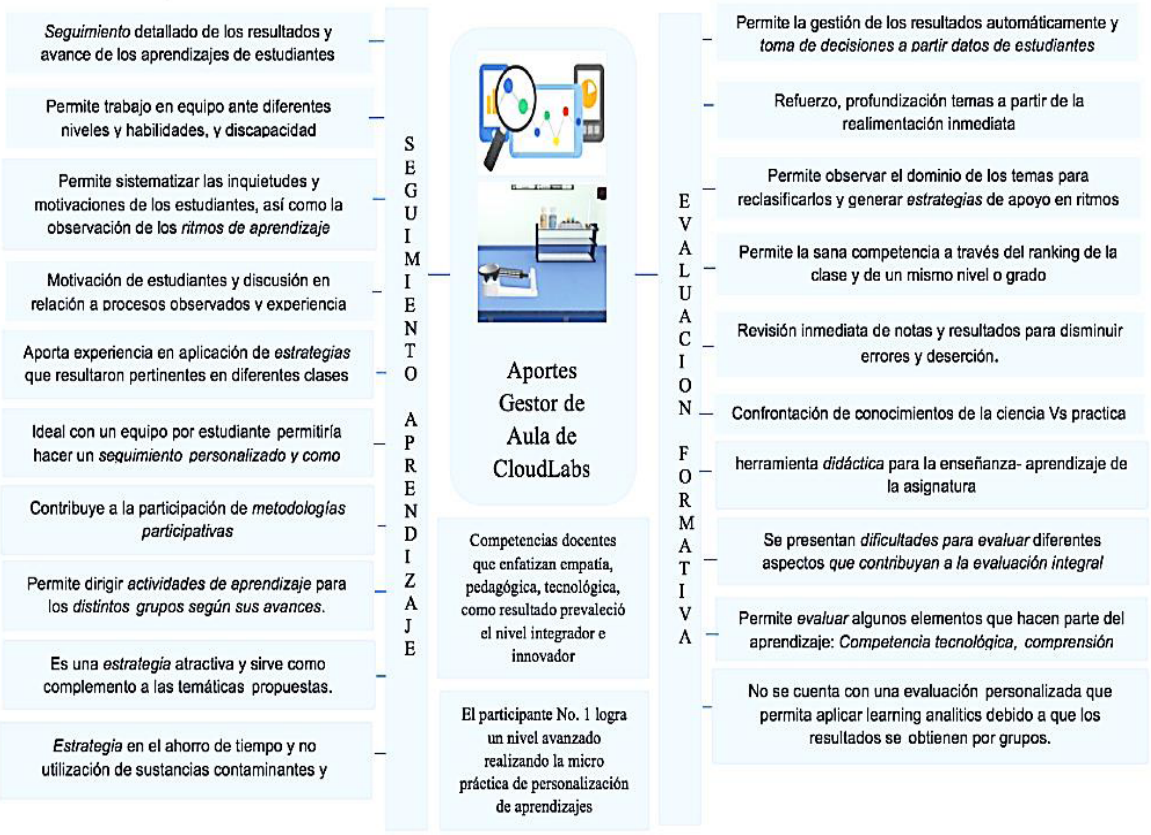

Figura 3. Aportes del gestor de aula. Fuente: elaboración propia. 
En relación con la micropráctica sobre Learning Analytics actividad 2, tema 2-, es de observar que el resultado fue logrado por el participante 1, ubicado en el nivel avanzado como caso de éxito Big Teacher, por el diseño de un itinerario personalizado de una clase con una complejidad baja, media y avanzada para sus estudiantes. El docente realizó las actividades en los tiempos dispuestos y superó las diferentes dificultades mediante estrategias y mecanismos utilizados. Las fases de esta micropráctica fueron:

- Observación: el docente utilizó su saber pedagógico para identificar las condiciones necesarias en aula, en busca de generar un ambiente de aprendizaje adecuado a las necesidades de los estudiantes, lo cual varía según el contexto sociocultural y la riqueza de sus interacciones. Por eso es importante el diagnóstico o evaluación de los conceptos previos no solo al inicio del proceso educativo, sino a lo largo de todo el desarrollo formativo, como lo explica el docente cuando afirma:

La labor docente en el proceso educativo es constante por la necesidad de retroalimentar en casi todo momento, y en gran medida de la habilidad o competencia del docente por observar todos los detalles, incluso aquellos que parecen sutiles a nuestra vista (Opinión de docente, registro de observación 1).

- Planificación: el docente trabajó dos semanas en el diseño de las clases, con tiempo adicional para su aplicación y observación de sus estudiantes. En esta fase se puede evidenciar la necesidad de desarrollar las competencias analíticas dado que, como lo afirma el docente, "planear diferentes actividades con un mismo eje temático es un reto, desde reunir los recursos dependiendo del tipo de estudiante que los abordará, hasta las herramientas TIC que se van a usar" (Opinión de docente, registro de observación 1).

- Aplicación: el docente puso en práctica la propuesta diseñada sin indicarles a los estudiantes que tenían diferentes actividades sobre un mismo tema. Hizo esto para promover el mejoramiento académico y comprender en qué momento del aprendizaje se encuentran:

En relación con el uso del gestor, después de sortear algunos inconvenientes, se pudo implementar en el desarrollo de varias prácticas, pero hubo dificultad para recibir por este medio los reportes generados por el CloudLabs. Se pudo identificar que el problema es con la red Aruba que tiene el colegio. Ante esta 
situación se optó por que enviaran los reportes a través del aula virtual de química en Schoology (Opinión de docente, registro de observación 1).

- Reflexión: después de tener los datos arrojados por el gestor de aula y el laboratorio virtual, el rol del docente se centra en mejorar los desempeños de los estudiantes a estados más avanzados. Por supuesto, son importantes las reflexiones que pueda hacer sobre su práctica, como una contribución a su aprendizaje y al mejoramiento del quehacer del docente:

Fue interesante realizar esta práctica. A pesar de abordar una temática que he trabajado durante varios años, utilicé nuevos recursos, la mayoría de ellos interactivos. Es complejo planear este tipo de estrategias por la cantidad de estudiantes que tenemos en las instituciones oficiales, incluyendo estudiantes con alguna discapacidad (Opinión de docente, registro de observación 1).

Parte de los retos en el desarrollo de esta actividad fue superar algunas limitaciones entre las que se encuentra el número de licencias de laboratorio virtual -solo 20 para el área de química-, lo que lleva a realizar las prácticas simuladas por parejas de estudiantes. Asimismo, el docente detectó limitaciones propias de la herramienta gestor de aula, al no contar con algunos servicios y otras soluciones existentes en el ámbito académico.

Por otro lado, resultaron interesantes para el proceso educativo las reflexiones docentes sobre las competencias a desarrollar e integrar en sus aulas. En ese orden de ideas, fue necesario determinar el nivel en el cual se encontraban los docentes respecto a las competencias MEN (2013), por lo que se procedió a aplicar al inicio del proyecto un diagnóstico enfocado en las competencias tecnológicas, pedagógicas y de gestión; competencias docentes.

A partir de la reflexión, los docentes opinaron sobre las competencias que deberían tener para una correcta integración de estas tecnologías al aula de clase. Como resultado se obtuvo que

- Para el docente las competencias se dividen en dos: la competencia tecnológica y la competencia para la integración de dicha herramienta a la práctica en el aula, las cuales es necesario implementar mediante la identificación de las necesidades, la caracterización del grupo, los objetivos y metodologías complementarias. 
- Resalta la importancia que tiene para las instituciones educativas entender la necesidad de integrar la parte administrativa, académica, convivencial y directiva entorno a las TIC.

A continuación, se presentan otras reflexiones de los docentes, específicamente sobre las competencias del MEN (2013).

\section{Competencia tecnológica}

Los docentes deben fortalecer el usoy, de ser posible, el desarrollo de objetos de aprendizaje que permitan a sus estudiantes aprender de manera comprensiva y significativa. Para el profesor resulta importante hacer uso de los recursos como el CloudLabs, tener más argumentos en el desarrollo de una cultura pedagógica mediada por TIC y fomentar la capacitación en las nuevas herramientas digitales para la analítica de datos.

\section{Competencia pedagógica}

Los docentes consideraron esta competencia como la más importante, porque dirige toda la estrategia implementada, con recursos digitales adecuados al nivel y pertinencia de los diferentes cursos de estudiantes.

\section{Competencias de gestión}

Es una competencia por desarrollar entre los docentes, no solo en una planeación de los contenidos temáticos, sino con los recursos tecnológicos y herramientas digitales necesarias. En su opinión, consiste en prever las posibles estrategias adicionales cuando se presenta una dificultad con esos recursos, como lo hacen al recibir un grupo que requiere una nivelación porque no tienen los prerrequisitos para abordar un eje temático planeado.

\section{Competencia investigativa}

En el campo educativo no hay una cultura de investigación en torno a Learning Analytics. Consideran que es una oportunidad de afrontar el reto de mejorar los ambientes de aprendizaje para que los estudiantes aprendan de manera comprensiva y significativa.

\section{Competencias a desarrollar}

Durante el proceso de investigación fue posible apreciar otras competencias que surgieron de la observación y de lo expresado por los docentes. Entre estas competencias está la analítica, como 
aquella que permite comprender y utilizar datos para mejorar la enseñanza y apoyar a sus estudiantes en el logro de sus objetivos de aprendizaje. También está la competencia colaborativa, entendida como la ayuda mutua surgida en la interacción entre los diferentes roles, donde el docente se define como facilitador $\mathrm{u}$ orientador del proceso pedagógico.

De igual manera, los docentes consideraron importantes las competencias como: la empatía, que facilita las relaciones, el direccionamiento y logro de los objetivos con sus estudiantes; el liderazgo, en el empoderamiento del proceso enseñanzaaprendizaje; y la administración del tiempo, ante el número de grupos de estudiantes y las diferentes labores académicas y administrativas a su cargo.

Otra competencia que surgió en el transcurso de la investigación fue la comunitaria, la cual, aparte de ser requerida por los docentes en las relaciones interpersonales con sus pares, personal directivo y administrativo, resulta importante al involucrar a las familias con el apoyo de la Guía alianza familia-escuela (SED, 2018a). No es una tarea fácil, pero se puede generar la cultura del seguimiento de los aprendizajes en los padres y apoyo escolar, con la ayuda de herramientas TIC como Edmodo y plataformas de notas.

Igualmente, surgió la competencia inteligencia emocional, como apoyo a la gestión de las propias emociones y delos estudiantes, desde las situaciones diarias a las que se ven enfrentados los docentes: los objetivos de enseñanza-aprendizaje, motivar a sus alumnos, dominio de grupos de 40 estudiantes en promedio, conflictos sociales e indisciplina. En la figura 4 se presenta la integración de las diferentes competencias detectadas durante la investigación.

Con estos resultados, se pudo mostrar que las herramientas de analítica de datos Learning Analytics en sinergia con el laboratorio virtual CloudLabs pueden ser implementadas en el proceso de aprendizaje y lograr unos efectos importantes en la práctica de aula. Los primeros resultados confirman los beneficios en relación con la personalización de aprendizajes y en la evaluación formativa por parte de los docentes.

Por su parte, los resultados posteriores son el reflejo de la importancia de una reflexión constante sobre la práctica pedagógica, que entienda al rol del docente como guía del proceso pedagógico 
COMPETENCIAS QUE

DESARROLLAN

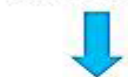

ANALÍTICA

COLABORADOR

FACILITADOR

ISTE (2017)
COMPETENCIAS (MEN, 2013)

TECNOLÓGICA

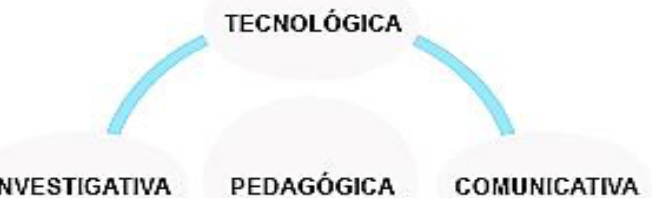

DE GESTIÓN

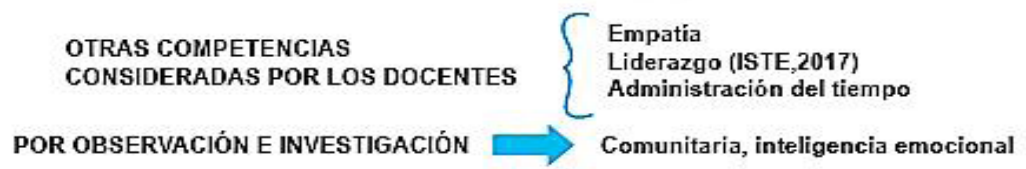

Figura 4. Competencias desarrolladas y emergentes. Fuente: elaboración propia.

y constructor de herramientas de aprendizaje tecnopedagógico para lograr los resultados de aprendizaje esperados. En este sentido, cobra importancia el desarrollo de las competencias desde diferentes niveles, pues, según Riaz y Khan (citados por Chiappe \& Cuesta, 2013), estas competencias conllevan "la toma de decisiones oportunas y acertadas, la comunicación asertiva, afectiva y efectiva, la solución de conflictos de manera creativa y exitosa, la cooperación y trabajo en equipo, todo ello dentro y fuera del aula de clases" (p. 505).

\section{Conclusiones}

Luego de la implementación del ambiente de aprendizaje para el desarrollo de la competencia analítica en los docentes, es posible afirmar que estas herramientas permiten hacer un seguimiento detallado de los resultados y avance de los estudiantes, ya que se puede ofrecer una realimentación inmediata a los estudiantes, refuerzo y profundización de temas al detectar las falencias en los diferentes ritmos de aprendizaje.

En respuesta a la pregunta que orientó la investigación, relacionada con la contribución a los docentes de ciencias naturales, en el seguimiento de los procesos de aprendizaje y evaluación 
formativa de los estudiantes, mediante la analítica de datos en un ambiente de aprendizaje b-learning, se puede afirmar que el gestor de los laboratorios, al organizar reportes sobre los avances de los estudiantes, permite al docente hacer un seguimiento en tiempo real sobre los diferentes estilos de aprendizaje y considerar cómo están aprendiendo, qué temas dominan o en cuál tienen dificultades, sus ritmos y limitaciones (Díaz \& Hernández, 2002). Por lo tanto, esto se convierte en un instrumento para la gestión de las estrategias didácticas, acordes a las necesidades de aprendizaje de cada uno de sus estudiantes. En términos de operatividad, el uso de estas dos herramientas dinamiza y flexibiliza la práctica real, en la medida que permite generar actividades interactivas, participativas y creativas adaptadas a los avances individuales.

Es importante tener en cuenta que la implementación de las tecnologías emergentes requiere del fortalecimiento de competencias de apoyo a los docentes y directivos, a saber: la competencia pedagógica para el manejo de sus clases; la tecnológica, para el manejo de estas tecnologías; para a partir del conocimiento de plataformas de analítica de datos, desarrollar la competencia analítica planteada por el ISTE (2017). Sin duda, la sinergia de estas competencias lleva al docente a ejercer una mejor gestión en su labor. De igual forma, el desarrollo tecnológico de herramientas de Learning Analytics requiere más investigación y adaptación al sector de la educación básica y media. Una recomendación para el futuro de estos desarrollos es invertir en el mejoramiento de las métricas de adaptabilidad, mediante sistemas dinámicos que han probado beneficios, pero que tienen un alto costo (Gauthier et al., 2015). Un ejemplo es el desarrollo de métricas y metodologías para cuantificar la adaptabilidad de sistemas de sistemas, o SoS Systems of Systems-.

Finalmente, para la consolidación de este tipo de investigaciones es necesario el apoyo de las instituciones educativas, en cuanto a tiempo, disposición, espacios y capacitación constante. También se requiere involucrar a las familias, a través del uso de las TIC, para el monitoreo del aprendizaje de los estudiantes en pos de lograr mejores resultados. Por eso, integrar las tecnologías emergentes en los colegios oficiales de Colombia requiere de una buena infraestructura con dotaciones de equipos y la oportunidad de que estos espacios estén disponibles para la comunidad educativa. 


\section{Referencias}

Amo, D., \& Rodríguez, S. (2017). Learning Analytics. La narración del aprendizaje a través de los datos. Barcelona: UOC.

Badia, A., Chumpitaz, L., Vargas, J., \& Suárez, G. (2016). La percepción de la utilidad de la tecnología conforma su uso para enseñar y aprender. REDIE. Revista Electrónica de Investigación Educativa, 8(3), 95105. Recuperado de https://www.redalyc.org/pdf/155/15547471007. pdf

Betancourt, V. (2004). La Cumbre Mundial Sobre la Sociedad de la Información: proceso y tema debatido. Asociación para el Progreso de las Comunicaciones. Recuperado de https://www.apc.org/sites/ default/files/wsis_process_ES.pdf:

Blikstein, P., \& Worsley, M. (2016). Multimodal Learning Analytics and Education Data Mining: using computational technologies to measure complex learning tasks. Journal of Learning Analytics, 3(2), 220238. http://dx.doi.org/10.18608/jla.2016.32.1

Chiappe, A., \& Cuesta, C. (2013). Fortalecimiento de las habilidades emocionales de los educadores: interacción en los ambientes virtuales. Educación y Educadores, 16(3), 503-524. Recuperado de https://www.redalyc.org/articulo.oa?id=83429830006

Chiappe, A., \& Rodríguez, L. (2017). Learning Analytics in 21st century education: a review. Ensaio: aval. pol. públ. Educ, 25(97), 971-999. https://doi.org/10.1590/So104-403620170025012

Concari, S. (2014). Tecnologías emergentes ¿̇cuáles usamos?. Lat. Am. J. Phys. Educ., 8(3), 494-503.

Congreso de Colombia. (2012). Ley estatutaria 1581 por la cual se dictan disposiciones generales para la protección de datos personales. Bogotá: Defensoría del pueblo. Recuperado de http://www. defensoria.gov.co/public/Normograma\%202013_html/Normas / Ley_1581_2012.pdf

Díaz, F., \& Hernández, G. (2002). Estrategias docentes para un aprendizaje significativo: una interpretación constructivista. Ciudad de México: McGraw Hill.

Esteve-Mon, F., Gisbert, M., \& Lázaro, J. (2016). La competencia digital de los futuros docentes: ¿cómo se ven los actuales estudiantes de educación?. Perspectiva Educacional, 55(2). Recuperado de http:// www.perspectivaeducacional.cl/index.php/peducacional/article/ view/412/207 
García, H. (2016). Uso de los laboratorios virtuales para la enseñanzaaprendizaje del concepto materia y sus propiedades en estudiantes de grado noveno. Manizales: Universidad Nacional de Colombia.

Gauthier, J., Miner, N., Wilson, M., Le, H., Kao, G., Melander, D., \& Vander Meer Jr, R. (2015). Quantitative adaptation analytics for assessing dynamic systems of systems. United States: LDRD final report.

Hernández, R., Fernández, C., \& Baptista, C. (2006). Metodología de la investigación. Ciudad de México: McGraw-Hill.

Hung, E. (20 de diciembre de 2015). El uso del Learning Analytics para la mejora de la calidad de los ambientes de aprendizaje. Recuperado de https://compartirpalabramaestra.org/actualidad/columnas/ el-uso-del-learning-analytics-para-la-mejora-de-la-calidad-de-losambientes-de-aprendizaje

ISTE. (2017). Estándares ISTE para docentes. Eduteka. Recuperado de http://eduteka.icesi.edu.co/pdfdir/iste-estandares-docentes-2017. pdf

ISTE. (2019). Estándares ISTE para los docentes. ISTE. Recuperado de https://www.iste.org/es/standards/for-educators

Lugo, C., \& González, L. (2018). La competencia analítica como herramienta para transformar el aula. Ruta Maestra, Santillana, (24), 127-132.

Ministerio de Educación Nacional. (2006). Fundamentos conceptuales. Bogotá: MEN. Recuperado de https://www.mineducacion.gov. co/1621/fo-article-299611.pdf

Ministerio de Educación Nacional. (2013). Competencias TIC para el desarrollo profesional docente. Bogotá: MEN.

Niño, M., \& Ariza, J. (2015). Herramienta de Learning Analytics para el proceso de aprendizaje en un aula virtual. Bogotá: Fundación Universitaria Los Libertadores.

Secretaría de Educación de Bogotá. (2017).Resolución 1944 Por medio de la cual se adopta la Política de Seguridad de la Información de la Secretaría de Educación del Distrito. Bogotá: SED. Recuperado de https://www.alcaldiabogota.gov.co/sisjur/normas/Norma1. jsp?i $=68201 \& \mathrm{dt}=\mathrm{S}$

Secretaría de Educación de Bogotá. (2018a). Guía metodológica para el fortalecimiento de la alianza familia-escuela. Bogotá: SED. Recuperado de https://www.educacionbogota.edu.co/archivos/ NOTICIAS/2018-/PW_Libro_Alianza_Familia_Escuela.pdf 
Secretaría de Educación de Bogotá. (2018b). Saber Digital. Recuperado de https://www.redacademica.edu.co/estrategias/saber-digital

Secretaría de Educación de Bogotá. (2018c). Resolución 1379 Por la cual se adopta la Política de Tratamiento de Datos Personales de la Secretaría de Educación del Distrito. Bogotá: SED. Recuperado de alcaldiabogota.gov.co/sisjur/normas/Norma1.jsp?i=81759\&dt=S

Shute, V., \& Rahimi, S. (2017). Review of computer_based assessment for learning in elementary and secondary education. Journal ComputerAssites Learning, 33(1), 19. doi:10.1111/jcal.12172

Siemens, G., \& Long, P. (2011). Penetrating the fog: Analytics in learning and education. Educause review, 46(5), 31-40. Recuperado de https://er.educause.edu/ /media/files/article-downloads/erm1151. pdf\%20

Unesco. (2013). Enfoque estratégico sobre las TIC en educación en América y el Caribe. Santiago de Chile: Oreal y Unesco.

Unesco. (2017). Aprendizaje personalizado. Ginebra: Unesco.

Wong, J., Baars, M., Koning de, B., Van der Zee, T., Davis, D., Khalil, M... Paas, F. (2019). Educational Theories and Learning Analytics: From Data to Knowledge. En D. Ifenthaler, D. Mah., \& J. Yau (Eds.), Utilizing Learning Analytics to Support Study Success (pp. 3-25). Suiza: Springer. 